\title{
THE USE OF THE OPHTHALMOSCOPE IN PRACTICE
}

\author{
By R. LINDSAY REA, M.D., F.R.C.S. \\ (Ophth. Surg., West End Hosp. Nervous Diseases; Surg., Western Ophth. Hosp., etc.)
}

The invention of the reflecting ophthalmoscope by von Helmholz in I85I opened wide horizons in medicine. When von Graefe for the first time saw the back of the eye with its nerve entrance and blood vessels, his cheeks reddened, and he called out excitedly, "Helmholz has unfolded to us a new world."

Of the many instruments of investigation produced in the practice of medicine, surely the ophthalmoscope takes premier place: so, for a short time, let us take up this instrument and consider its use. The electric ophthalmoscope is the most common form used. One of the cheapest is the May-Wappler, the battery of which is the same as that used in the Ever-Ready pocket torch. However, a first-class instrument such as the Hamblin or that made by Davis Keeler is a sound investment, and is a real pleasure to use. An oculist cannot do without the older pattern (the Morton), which is used for indirect ophthalmoscopy, but the physician or neurologist need only use the direct ophthalmoscope.

\section{Procedure.}

Adults.-To use the ophthalmoscope correctly the examiner seats himself beside his patient, not in front. On no account should he use his legs as a vice wherewith to hold his patient. The patient's head should be slightly inclined towards the examiner, whose head is inclined slightly towards the patient, with a distance of not much more than the thickness of the ophthalmoscope between the foreheads. If the position is correct the ophthalmoscope will be held vertical and not at $45^{\circ}$. It is not necessary to hold the patient by the hair of his head; every student seems to be taught this method at hospital. What a surprise is in store for him if he takes hold of the hair of a lady with a newly acquired "perm" or set. How she resents the man who carelessly disturbs it. In fact, such a patient and all her friends are lost to him at one stroke. Therefore if one has learnt the habit of holding a patient's hair, the habit should be unlearnt as quickly as possible.

To dilate the pupil in order to get better illumination, use a drop of a watery solution of homatropine and cocaine of each 2 per cent. In elderly people this should always be followed by a drop of $\frac{1}{2}$ per cent eserine at the conclusion of the examination. However, experience teaches one that a mydriatic is not always necessary, but there is an art in overcoming the pupillary reflex in the undilated eye. Remember there is a rheostat on the ophthalmoscope which regulates the amount of light emitted: also a slide which produces a small round uniform beam of light on pulling out the optical part away from the handle. Direct the light not straight into the pupil, but from the temporal side, so that a modified beam of light will fall on the disc area and not on the macula. If the light first falls on the macula the pupillary reflex contracts the pupil and little or nothing will be seen. Gradually open up the rheostat; as the eye soon becomes accustomed to the light the pupil remains open and a full investigation can be made. Sometimes the beginner has difficulty in finding the disc. All he has to do is to look at one large vessel and follow it up or down and this will lead him to the papilla. This found, approach the macular area slowly. This lies on the temporal side of the disc. Again reduce the amount of light for a moment until the faintly pigmented macula with a small bright reflex spot in the centre is seen. Gradually open up the rheostat. The remainder of the fundus is not nearly so sensitive to light as the macula, and so can be easily seen.

Children.-One is called upon at times to examine a baby's eye without an anaesthetic. The best time to do this will be during its sleep. Approach quietly and without rattling the side of the cot; place the thumb of the disengaged hand gently on the upper lid and wait until there is no movement of the head. Slowly lift the lid and examine the eye with the ophthalmoscope with a reduced beam of light. If the light is gradually increased it will be found that this does not waken the baby. A mydriatic is not necessary as a rule.

If, however, the baby's eye is to be examined under an anaesthetic, it should be dilated with $\frac{1}{2}$ per cent atropine, one drop the previous night, and one drop an hour before examination. 


\section{Normal Fundus.}

Get to know the appearance of the normal fundus before attempting to recognise pathological conditions. Look at Plate I (The Fundus of the Normal Eye). On the right is the optic disc with some choroidal pigment around its edge. This may form a complete ring at times. The surface of the optic papilla is paler than the surrounding retina. In the centre of the papilla is the normal physiological pit which is much paler than the surrounding surface. At the bottom of the pit the lamina cribrosa is seen with more or less distinctness. These are the fibres of the sclera through which bundles of the optic nerve pass. The arteries are light in colour, and are seen emerging from the depth of the physiological pit. The veins are darker. To the left is the normally pigmented macula and usually with a bright reflex spot in the centre. Carefully examine where the arteries cross the veins and note that they do not normally kink each other as they cross.

\section{Abnormal Fundus.}

We are now in a position to understand the appearance of departures from the normal. Plate 2 (Papilloedema). The optic disc red and swollen, is seen protruding into the vitreous. The edge of the disc is obscured by oedema, and fine haemorrhages on and around the disc are present. It should be noted that this disturbance is confined to the region of the disc, and except for swollen veins the remainder of the fundus is normal. Rarely a fan-shaped appearance between the disc and the macula is observed. Such an appearance of the fundus is the opposite to the condition known as arteriosclerotic retinopathy, where, in addition to the swollen disc small flame-shaped haemorrhages and white spots are found all over the fundus. In the retinitis of diabetes, known as hypertensive neuro-retinopathy (see Plate 5 -Retinitis in Diabetes), the haemorrhages are larger, being deeper or, if subhyaloid, have the shape of a segment of a circle, the upper edge of which is hiorizontal. The exudates may be of the cotton-wool variety, or waxy with sharp-cut edges. These, too, are scattered over the fundus. In renal retinopathy the exudates may form a star-shaped figure around the macula.

The degree of papilloedema due to intracranial pressure can be measured by the ophthalmoscope. In order to measure accurately the amount of swelling of the nerve head the student must learn to relax voluntarily his own accommodation. To prove that he can do so he should be able to see instantly the double image of a pencil held eight inches from his face while looking at a wall or ceiling beyond. The same effort must be made while looking at the surface of the disc. The examiner, keeping his accommodation in abeyance and focussing the light on the vessels of the surface of the disc, rotates the convex lenses to the highest number with which he can still see distinctly the highest vessel. Suppose he then rotates a higher convex lens before his eye the edge of the vessel will appear slightly blurred. He will now return to the lens which gave him a distinct view of the highest vessel on the surface. Noting the number of this lens he will now direct his attention to the vessels of the fundus situated some distance from the edge of the disc. To see these sharply he must reduce the number of the lens in the ophthalmoscope and stop at that number which gives the clearest view of these vessels. Subtracting the latter number from the former gives the height of the papilloedema in diopters. It should be remembered that three diopters are equal to one millimetre of swelling.

When the papilloedema has subsided the edge of the disc remains blurred, the physiological pit is filled in, and the colour of the disc often fades to gray. This is known as consecutive optic atrophy. If the pressure on the nerve head has lasted three months, there is great danger of the sight of the eye being lost. Hence always the urgency to decrease the papilloedema by decompression or other means.

Compare Plate 4, which illustrates Primary Optic Atrophy, with Plate 2-Papilloedema. In primary optic atrophy the disc is white, the edge is sharply defined, and the physiological pit, if present, is not filled in. The vessels may be reduced in size, but often are not. When atrophy follows an inflammatory change in the nerve and adjoining retina, the condition is referred to as secondary optic atrophy, but when the atrophy follows a papilloedema from raised intracranial pressure, it is known as consecutive optic atrophy. If you remember these terms, it will always save confusion of thought.

In both secondary and consecutive optic atrophy the vessels of the fundus may show fine white lines accompanying them in their course.

Finally, it must be pointed out that a frequent mistake made by students is to diagnose a case of opaque nerve fibres as papilloedema. Plate 5 (opaque Nerve Fibres) illustrates a 


\section{Subtitles to Plates}

\section{Plate 1. The Normal Fundus.}

On the right is the optic disc with some choroidal pigment around its edge. The surface of the optic papilla is paler in colour than the surrounding retina. In the centre of the papilla is the normal physiological pit which is much paler in colour than the surrounding surface, and, at the bottom of the papilla, the lamina cribosa is faintly seen. The arteries are lighter in colour and are seen emerging from the depths of the physiological pit. The veins are darker in colour. To the left is the normally pigmented macula. Note that where the arteries cross the veins there is no kinking.

\section{Plate 2. Papilloedema.}

Papilloedema or "choked disc" due to raised intracranial tension. In this instance the papilloedema was due to a frontal lobe tumour.

\section{Plate 3. Retinitis in Diabetes, or Hypertensive Neuro-Retinopathy (Fischberg).}

In this condition haemorrhages and exudates are scattered over a wide area of the retina. The haemorrhages may be found in the deeper layers of the retina and are then somewhat circular in shape, but large subhyaloid haemorrhages are commonly found. These have the shape of a segment of a circle, the upper edge of which is horizontal. The exudates may be either of the cotton-wool variety or waxy with sharp-cut edges: they, too, are scattered over the fundus and do not form a star-shaped figure at the macula.

\section{Plate 4. Primary Optic Atrophy.}

The optic disc is greyish-white with sharp edge and normal choroidal ring. Frequently the colour of the disc in primary optic atrophy is of a marble whiteness. The physiological pit is present, and the size of the vessels is normal or slightly reduced in calibre. The remainder of the fundus appears normal. When pigment is present in the retina surrounding the disc due to inflammatory changes it is never so black nor definitely outlined as is the normal choroidal pigment.

\section{Plate 5. Opaque Nerve Fibres.}

This peculiar appearance, which in many cases strongly suggests a papilloedema, is due to the presence of the myelin sheath of the nerves in the retina which normally ends behind the lamina cribrosa. Rarely these white patches are seen separated some distance from the optic disc, but when, as in this picture, the white area more or less surrounds the optic edge the retinal vessels are seen to disappear and appear again at the edge of this area, giving the impression that the vessels are obscured by oedematous exudates. The medullary nerve-sheath areas are always characterised by the brushlike extremities.

From Lindsay Rea's Neuro-Ophthalmology by kind permission of Messrs. W. Heinemann, Publishers. 


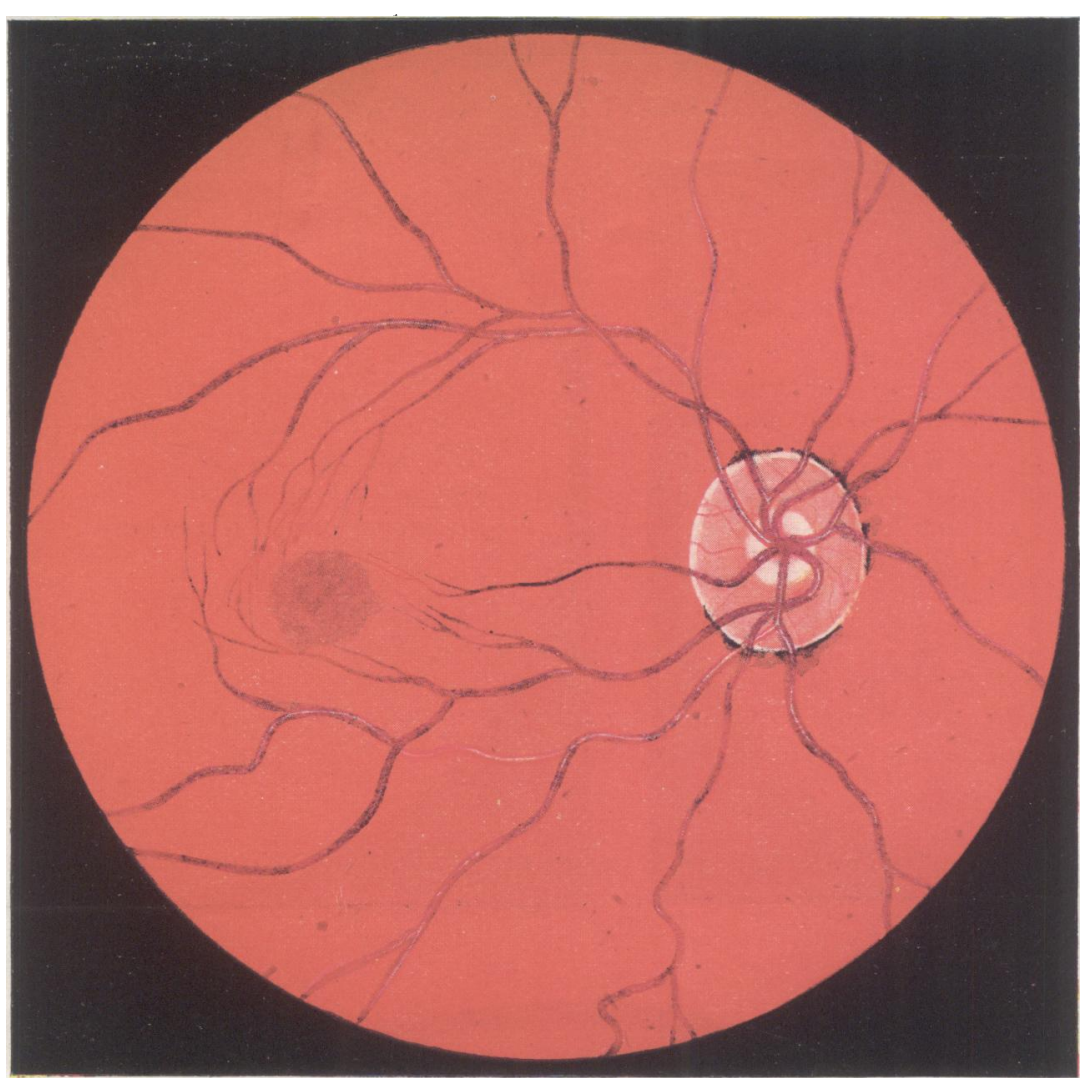

Plate r.-The Normal Fundus.

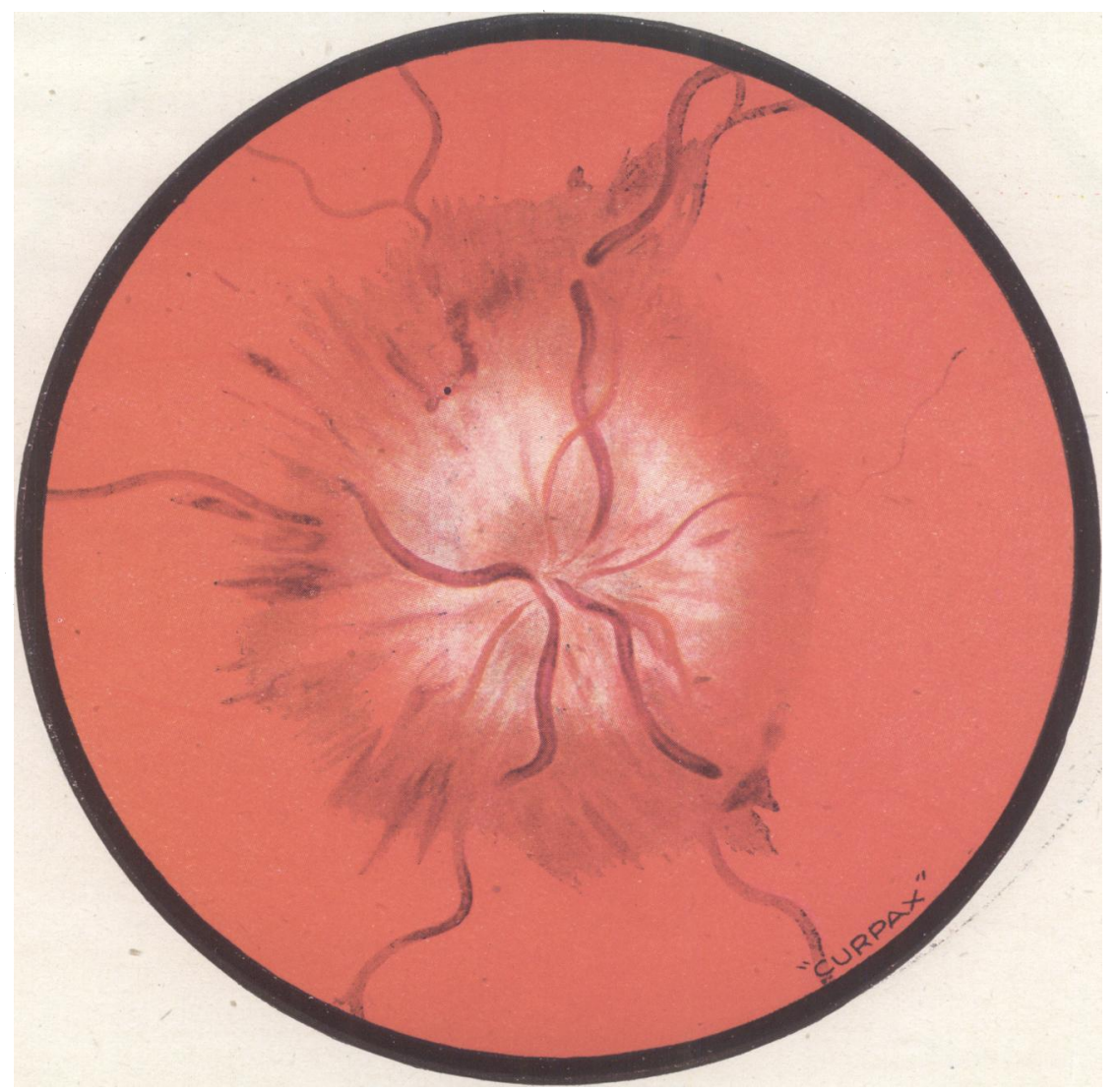

Plate 2.-Papilloedema. 


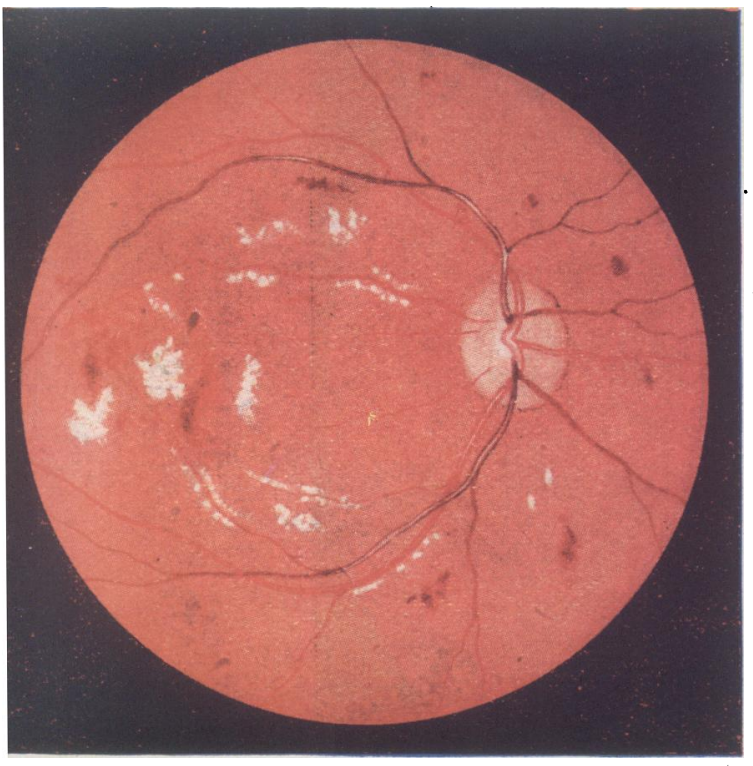

Plate 3.--Retinitis in Diabetes.

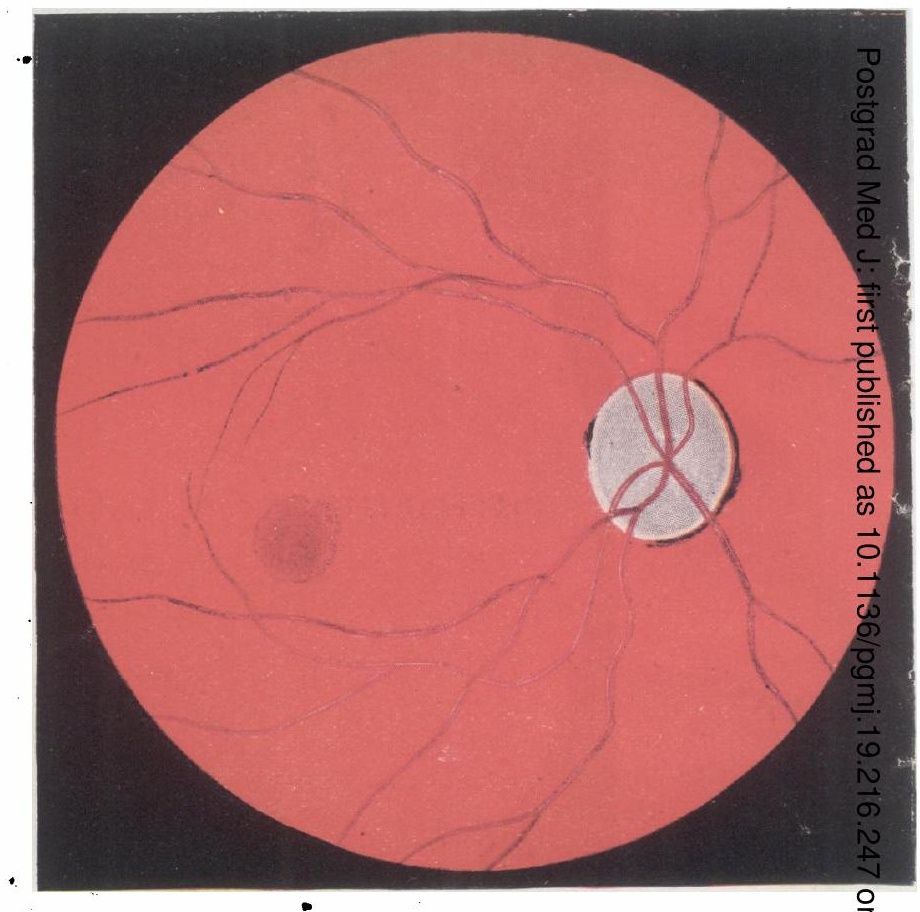

Plate 4.-Primary Optic Atrophy.

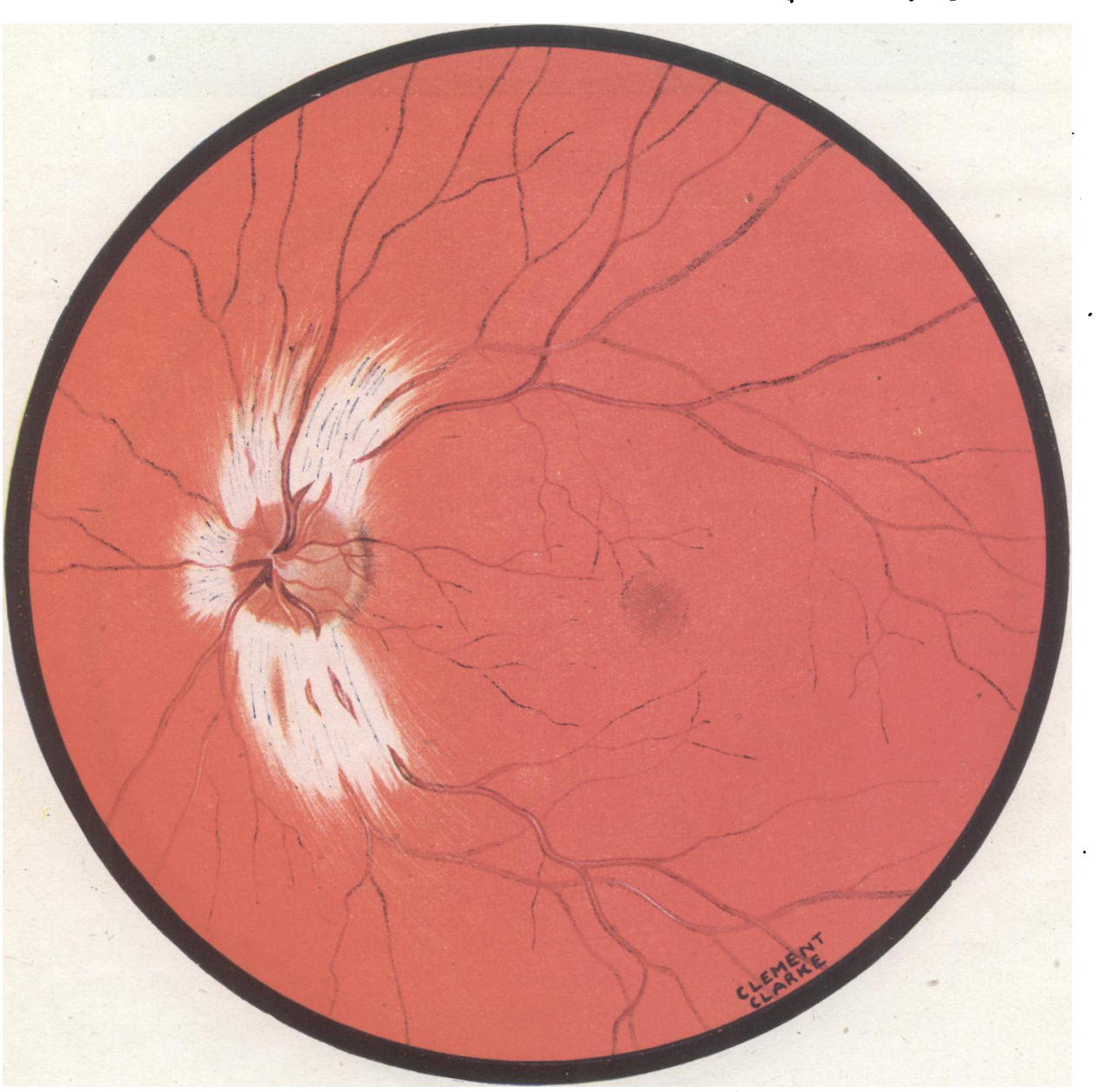

Plate 5.-Opaque Nerve Fibres. 
drawing taken from life. The optic nerve has become medullated beyond the disc, and when these medullated fibres are present the disc loses its sharp edge and the vessels disappear and reappear from the mass of white tissue so that there is a very definite resemblance to papilloedema. It will be noticed, however, that the surface of the nerve head is not elevated, and that the white area is not uniformly continued around the disc. Also there is an entire absence of oedematous or haemorrhagic change on or around this area. The veins, too, are not enlarged.

Let me repeat once more, learn to examine the normal fundus first before proceeding to the pathological.

\title{
OPHTHALMIC EMERGENCIES IN GENERAL PRACTICE
}

\author{
By G. G. PENMAN, M.A., M.D.(Cantab.), F.R.C.S. \\ (Ophth. Surg., O.P., St. Thomas's Hosp.; Surg., Royal Westminster Ophth. Hosp., etc.)
}

In an article of this type it is impossible to deal even shortly with all the conditions which might come under this head, and I have therefore excluded all conditions (e.g. lids), which are not entirely ocular, and those where early treatment is not of prime importance.

The importance of taking a careful history in all cases cannot be over-emphasised, especially when a foreign body is suspected.

Examination' must be made in a good light with adequate magnification. If there is much blepharospasm, 4 per cent cocaine may be instilled, or in a child a short general anaesthetic may occasionally be necessary.

In doubtful cases, it should be remembered that the use of atropine in patients under forty is practically always safe, and often indicated. Over forty there is always the danger of precipitating an attack of glaucoma in a subject with a tendency that way, or in cases where glaucoma is present, but not diagnosed, of making the condition infinitely worse.

\section{Foreign Bodies.}

\section{Injuries}

(a) Conjunctival.-Foreign bodies, usually tiny pieces of grit, are a frequent source of trouble. The most common position is in the upper fornix, often about 2 or $3 \mathrm{~mm}$. from the lid margin. Sometimes the foreign body is at the very apex of the fornix, so that when the lid is ordinarily everted it cannot be seen. If, with the lid still everted, the eye is pressed on from above, the remainder of the conjunctiva will roll out in a fold, bearing the foreign body with it. When found, the foreign body may be removed with a piece of sterile cotton-wool, or the corner of a clean handkerchief. If very adherent it must be picked out like a corneal foreign body (q.v.), The eye should be irrigated with saline for a day or two after.

(b) Corneal.-As a rule these need a spud, or even a needle, to move them. The eye must be thoroughly cocainised. One of the commonest foreign bodies found on the cornea is a black speck from an emery wheel. It usually embeds itself firmly in the cornea, and when the main portion has been removed leaves a little brown ring, which should also be cleared as much as possible, though care must be taken not to cause more harm to the eye by deep digging, causing subsequent scarring, than would be the case if a little of the ring were left. The eye should be irrigated with saline after the removal of the foreign body, and drops of atropine, I per cent instilled, and where there has been much digging, a pad and bandage applied.

(c) Intra-ocular.-See" perforating injuries.

\section{Abrasions. -}

Abrasions of the cornea are common. The eye is injected and watery, and the patient usually thinks that a foreign body is present. The cornea will often appear quite normal on casual inspection, but staining with fluorescin will show a bright green area where the cornea has been denuded of epithelium.

Treatment.--Irrigation with warm saline, and immobilisation of the lids with pad and bandage. Where a large area is affected, or there is any sign of infection (showing as cloudiness of the cornea in and round the wound), atropine I per cent may be used. These abrasions are often extremely painful, and sedatives may be necessary. Sometimes after months or years the epitholium peels off again in the same place, and symptoms recur. 